\title{
Research Paper \\ Relationship of Somatotype With Static, Semi-dynamic and Dynamic Balance of Adolescent, Young and Middle-aged Women
}

\author{
*Mahbubeh Keivan ${ }^{1}$ (1), Heydar Sadeghi ${ }^{2}$ \\ 1. Department of Sports Biomechanics, Faculty of Physical Education and Sports Science, Islamic Azad University Central Tehran Branch, Tehran, Iran. \\ 2. Department of Biomechanics and Sport Injuries, Faculty of Physical Education and Sports Sciences, Kharazmi University, Tehran, Iran.
}

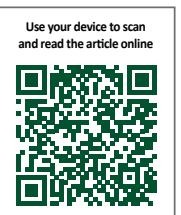

Cittation: Keivan M, Sadeghi H. [Relationship of Somatotype with Static, Semi-Dynamic and Dynamic Balance of Adolescent, Young and Middle-Aged Women (Persian)]. Journal of Sport Biomechanics. 2019; 4(4):42-53. https://oi.org/10.32598/ biomechanics.4.4.42

https://doi.org/10.32598/biomechanics.4.4.42

Keywords:

Somatotype, Static balance, Semi-dynamic balance, Dynamic balance

\section{ABSTRACT}

Objective Somatotype is a way of describing the human body shape and composition which can affect postural control. The aim of this study was to evaluate the relationship of somatotype with static, semidynamic and dynamic balances in adolescent, young and middle-aged women.

Methods Participants were 140 women aged $12-50$ years (Mean \pm SD of age $=26.45 \pm 10.94$ ) with no skeletal abnormalities, lower extremity pain and injury, vestibular and neuromuscular injury. Their anthropometric characteristics were measured and then they assigned into three groups of ectomorph $(n=46)$, mesomorph $(n=45)$ and endomorph $(n=49)$ based on the heath-carter method. The Y Balance Test (YBT) was used for assessment of dynamic balance, and for semi-dynamic and static balance measurement, the Balance Error Scoring System (BESS) was used. Descriptive statistics was used to describe Mean \pm SD of age, height, weight, number of errors under BESS, and the reach distance in three directions under YBT. To determine the relationship of somatotype with static, semi-dynamic and dynamic balance, Chi-square test was performed considering a significance level of $\mathrm{P} \leq 0.05$.

Results Mesomorphic women had better static and semi-dynamic balance compared to the ectomorph and endomorph groups. In anterior direction under YBT, ectomorph, mesomorph and endomorph groups had the highest reach. In posteromedial direction, although endomorphs had the poorest performance, the result was not significant. In posterolateral direction, endomorphs had also the poorest performance. Conclusion Better performance of mesomorphs indicated their better muscle strength and structure which are useful for joint stability and postural control. Moreover, the better postural control of ectomorphs compared to endomorphs shows that the mass distribution of more than the height of the center of gravity until the base of support which is one of the main determinants of stability, is probably the factor that differentiated their postural control.

\section{Extended Abstract}

\section{Introduction}

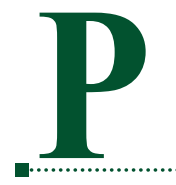

ostural control is a complex process, involving the center of gravity maintenance within the base of support. Moreover, it requires the co-ordination of the somatosensory, visual, and vestibular systems [1]. The information provided by these three systems is executed by the Central Nervous System (CNS) and integrated in the form of an appropriate motor response to maintain body balance. Balance is actively controlled by the CNS, which predicts the perturbations of whole body or trunk movements. A key feature of balance is stability.

* Corresponding Author:

Mahbubeh Keivan, M.A.

Address: Department of Sports Biomechanics, Faculty of Physical Education and Sports Science, Islamic Azad University Central Tehran Branch, Tehran, Iran.

Tel: +98 (939) 6595772

E-Mail:m.keivan2@yahoo.com 
Stability is related to motor control resistance and a smooth state without changing in response to a perturbation or oscillations creating perturbations. Typically, if the posture returns to its original state during the perturbation, it is considered as stable. Somatotype is a means of describing human body shape and condition in addition to height and weight; it can affect postural control [10-12]. Somatotype is also used to determine the body types most exposed to various diseases. Studies have examined the relationship between body type and health status [14-16] and physical activity $[15,17,18]$. Previous studies have reported that postural control is different in different somatotypes; however, they only examined the effect of somatotype on static or dynamic control in one age group. Thus, we examined the relationship between somatotype and static, semi-dynamic, and dynamic postural controls in adolescent, young, and middle-aged females.

\section{Participants and Methods}

A total of 140 females aged 12-50 years (Mean \pm SD of age $=26.45 \pm 10.94 \mathrm{y}$ ) with no skeletal abnormalities, lower extremity pain, and injury, as well as vestibular and neuromuscular injury were included in the study. The study participants' anthropometric characteristics were measured. Then, they were classified into three groups of ectomorph

Table 1. Postural control measurement results based on somatotype groups

\begin{tabular}{|c|c|c|c|c|c|c|}
\hline \multirow[b]{2}{*}{ Balance } & \multirow[b]{2}{*}{ Number of Errors } & Frequency & \multicolumn{3}{|c|}{ Group } & \multirow[b]{2}{*}{ Total } \\
\hline & & $\begin{array}{l}\text { Frequency Per- } \\
\text { centage }\end{array}$ & Ectomorph & Mesomorph & Endomorph & \\
\hline \multirow{15}{*}{ Static } & & $\mathrm{N}$ & 13 & 21 & 9 & 43 \\
\hline & $<3$ & $\%$ & 28.3 & 46.7 & 18.4 & 30.7 \\
\hline & & $\mathrm{N}$ & 10 & 10 & 7 & 27 \\
\hline & $3-6$ & $\%$ & 21.7 & 22.2 & 14.3 & 19.3 \\
\hline & & $\mathrm{N}$ & 4 & 2 & 7 & 13 \\
\hline & $6-9$ & & & & & \\
\hline & & $\%$ & 8.7 & 4.4 & 14.3 & 9.3 \\
\hline & & $\mathrm{N}$ & 9 & 2 & 9 & 20 \\
\hline & $9-12$ & & & & & \\
\hline & & $\%$ & 19.6 & 4.4 & 18.4 & 14.3 \\
\hline & & $\mathrm{N}$ & 5 & 6 & 9 & 20 \\
\hline & $12-15$ & & & & & \\
\hline & & $\%$ & 10.9 & 13.3 & 18.4 & 14.3 \\
\hline & & $\mathrm{N}$ & 5 & 4 & 8 & 17 \\
\hline & $>15$ & $\%$ & 10.9 & 8.9 & 16.3 & 12.1 \\
\hline \multirow{14}{*}{ Semi-dynamic } & & $\mathrm{N}$ & 46 & 45 & 49 & 140 \\
\hline & Toldal & $\%$ & 100.0 & 100.0 & 100.0 & 100.0 \\
\hline & & $\mathrm{N}$ & 20 & 30 & 18 & 68 \\
\hline & $<10$ & & & & & \\
\hline & & $\%$ & 43.5 & 66.7 & 36.7 & 48.6 \\
\hline & & $\mathrm{N}$ & 20 & 10 & 22 & 52 \\
\hline & $10-\angle 0$ & $\%$ & 43.5 & 22.2 & 44.9 & 371 \\
\hline & & $\mathrm{N}$ & 4 & 5 & 7 & 16 \\
\hline & $20-30$ & & & & & \\
\hline & & $\%$ & 8.7 & 11.1 & 14.3 & 11.4 \\
\hline & & $\mathrm{N}$ & 2 & 0 & 2 & 4 \\
\hline & $>30$ & $\%$ & 4.3 & 0.0 & 4.1 & 2.9 \\
\hline & & $\mathrm{N}$ & 46 & 45 & 49 & 140 \\
\hline & & $\%$ & 100.0 & 100.0 & 100.0 & 100.0 \\
\hline
\end{tabular}


$(n=46)$, mesomorph $(n=45)$, and endomorph $(n=49)$ based on the heath-carter method. Somatotype was determined based on the dominant component, i.e. $\geq 1.5$ units of difference from the other two components [13]. After determining the somatotype, the Y Balance Test (YBT) was performed to evaluate the study subjects' dynamic control.

\section{Results}

One-way Analysis of Variance (ANOVA) results suggested a significant difference in the mean static, semi-dynamic, and dynamic balance scores between the three groups of an ectomorph, mesomorph, and endomorph. Table 1 presents the postural control measurement results of the somatotype group. In total, $46.7 \%, 28.3 \%$, and $18.4 \%$ of the mesomorphs, ectomorphs, and endomorphs had the lowest error $<3$ in static postural control, respectively. This was also observed in the next category (3-6 errors). Regarding the highest error rate $>15$, only $8.9 \%$ of the mesomorphs had $>15$ errors, followed by ectomorphs with $10.9 \%$, and endomorphs with $16.3 \%$. In terms of semi-dynamic balance, a high percentage of mesomorph subjects $(66.7 \%)$ gained the lowest error rate $<10$, followed by $43.5 \%$ of ectomorphs, and $36.7 \%$ of endomorphs (Table 1 ).

\section{Discussion}

The current study investigated the relationship between somatotype, and static, semi-dynamic, and dynamic balance. The obtained results suggested a significant difference in the static, semi-dynamic, and dynamic balance between three ectomorph, mesomorph and endomorph female groups. The mean static balance (i.e. the number of errors in the Balance Error Scoring System test) in the mesomorph group (6.66) was significantly higher than the ectomorph (8.64) and endomorph (10.22) groups. Chi-square test results revealed that the mesomorph group indicated the best, and endomorphs demonstrated the poorest performance in static balance. This finding is consistent with those of Lee et al. [12] who reported that mesomorphic girls had substantially better one-sided static stability, compared to endomorphic and ectomorphic girls; they also reported the somatotype components effects on the postural stability in young girls.

The achieved data highlighted that the mesomorphic subjects had a better balance performance, while the performance of endomorphs was poor. Muscle strength and structure are useful for joint stability and postural control; the might be the main reason for the better performance of mesomorphs in our study (previous studies have supported the same finding) [11, 12, 29]. Unlike some previous studies, the ectomorphs in our study had better postural control than endomorphs. Although the high height-to-weight ratio is a characteristic of ectomorphs, the Mean \pm SD height of these subjects $(1.59 \pm 5.92)$ was not significantly different from that of endomorphs $(1.59 \pm 6.35)$; however, the difference between the two groups was significant in weight. Therefore, the height of the center of gravity to the base of support, which is one of the main determinants of stability, has not been increased; the mass distribution was probably the factor that differentiated the postural control of ectomorphs and endomorphs. Studies have documented that obese people with an asymmetric distribution of fat, especially in the abdomen, are more prone to fall [8].

\section{Conclusion}

The mesomorphic component of somatotype was associated with postural control. Besides, the endomorphic component impaired balance more than the ectomorphic component. Comparing these findings with those of other studies revealed the necessity for further investigations regarding the postural control of three somatotypes.

\section{Ethical Considerations}

\section{Compliance with ethical guidelines}

Prior to the study, a written informed consent was signed by the subjects or their parents. They were assured of the confidentiality of their information and were free to leave the study at any time.

\section{Funding}

The present paper was extracted from the MA thesis of the first author, Mahbubeh Keivan, Department of Sports Biomechanics, Faculty of Physical Education and Sports Science, Islamic Azad University Central Tehran Branch, Tehran.

\section{Authors' contributions}

Conceptualization, methodology, software, validation, formal analysis, investigation, resources, data curation, writing - original draft preparation, writing - review \& editing, visualization: Mahbubeh Keivan; Supervision, project administration: Heydar Sadeghi.

\section{Conflicts of interest}

The authors declared no conflict of interest. 


\title{
ارتباط تيڤ بدنى با كتترل ياسجر ايستا، نيمهيويا و يوياى زنان نوجوان، جوان و ميانسال
}

\author{
"محبوبه كيوان' (ه) حيدر صادقى' \\ ا. كروه بيومكانيك ورزشى، دانشكده تربيت بدنى و علوم ورزشى، دانشكاه آزاد اسلامى واحد تهران مركزي، تهران، ايران.

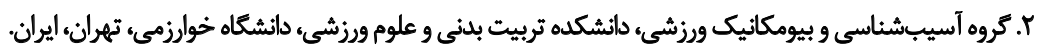

\begin{abstract}
حكيد

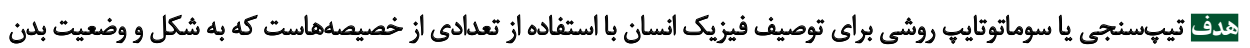

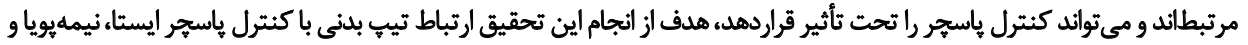

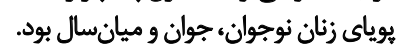

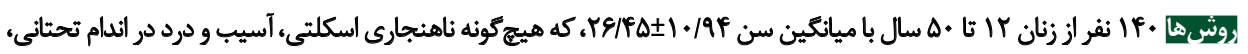

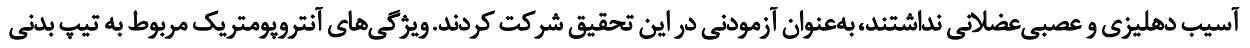

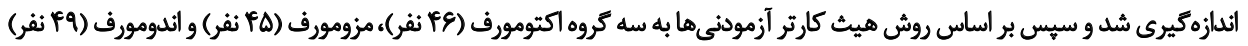

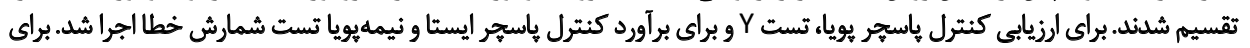

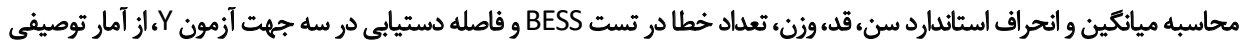

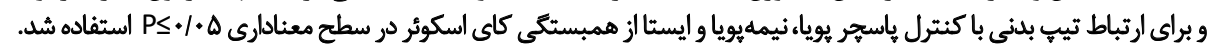

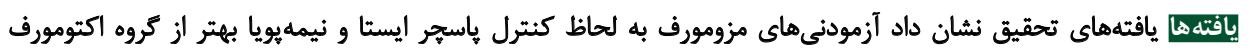

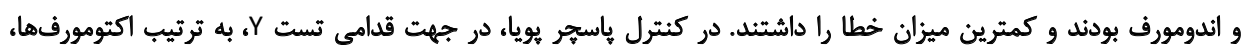

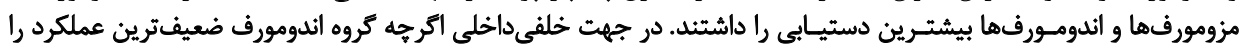

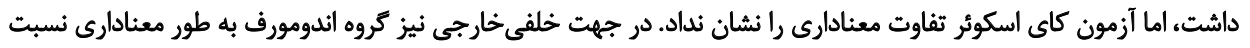

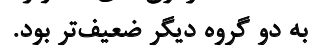

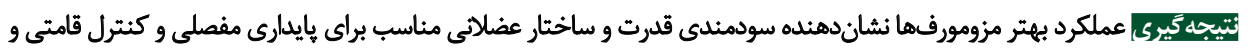

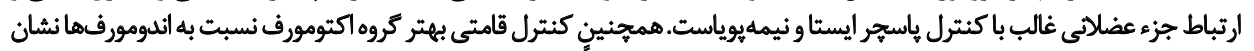

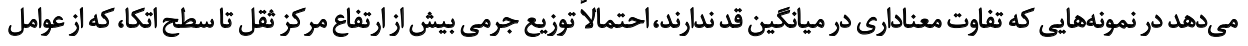

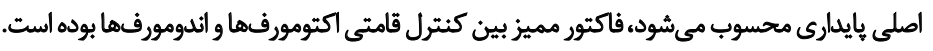

اطلاعات مقاله: - الم

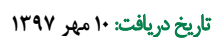

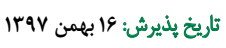

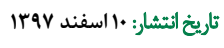

سيستم عصبى مركزى كه اغتشاشات ناشى از حركات كل بدن يا

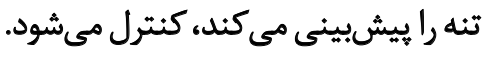

datios

يك ويرُّى اساسى توازن بايدارى قامتى است. يايدارى قامتى

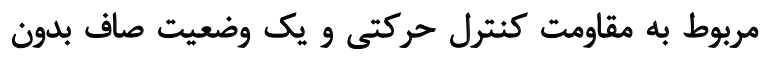

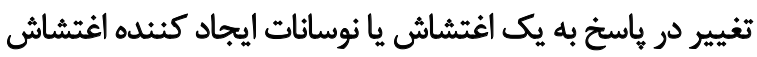

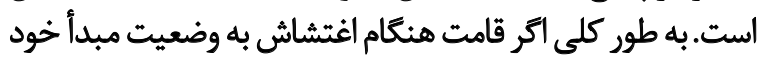

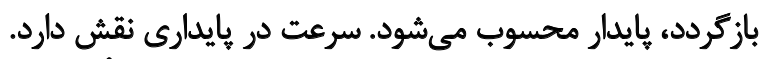

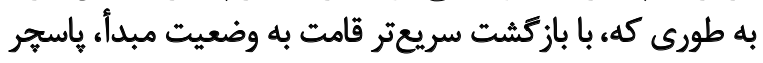

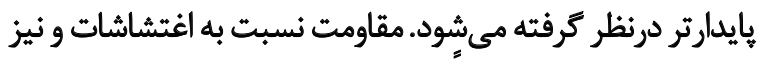

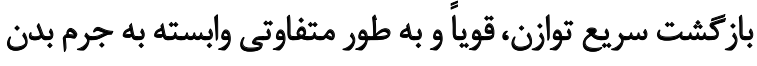

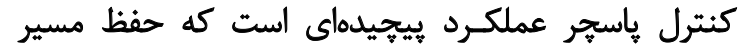

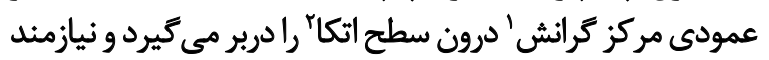

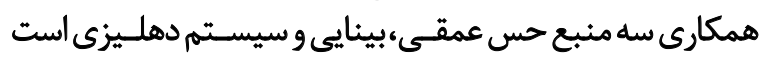

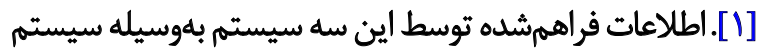

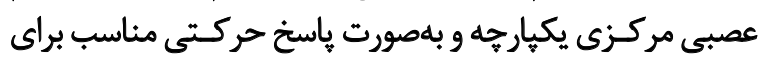

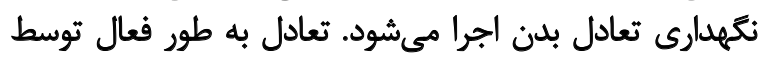

\section{Center of Gravity}

2. Base of Support 
مستلزم صرف انرري براى جابهجايى است. مربيان تربيت بدنى نيز

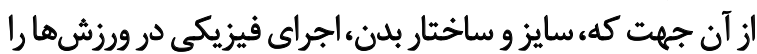

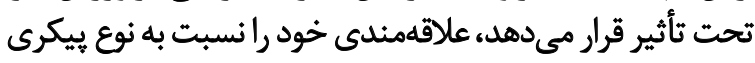

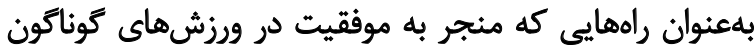

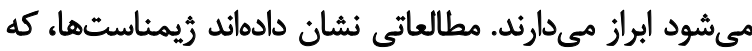

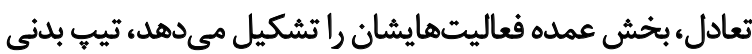

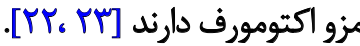

برخى كزارشات حاكى از آن است كه تفاوت عمده در طول

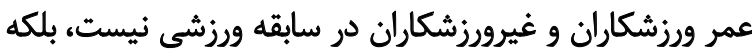

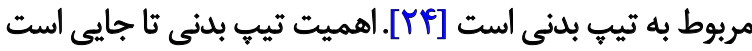

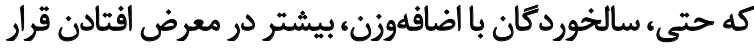

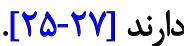

در تحقيق بلبليان آزمودنىهاى سالمندى كه فعال بوديند

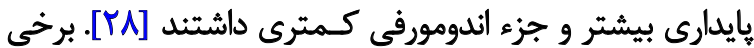

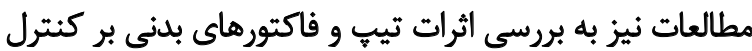

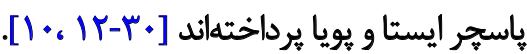

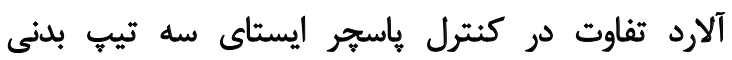

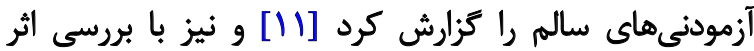

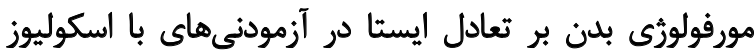

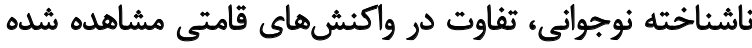

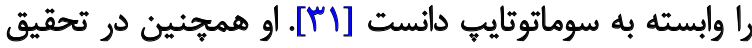

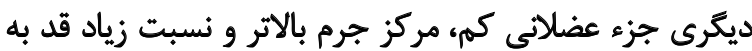

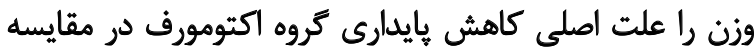

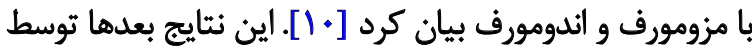

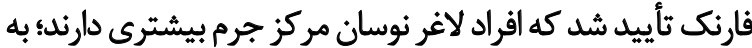

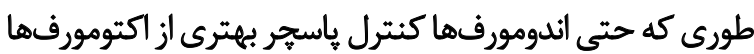

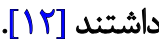

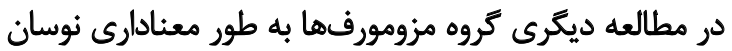

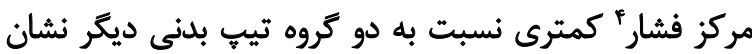

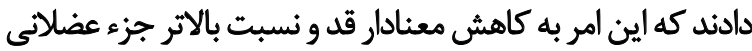

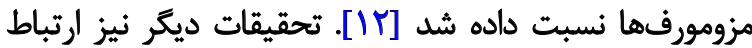

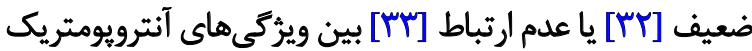

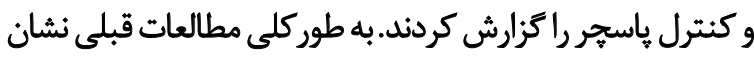

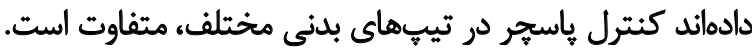

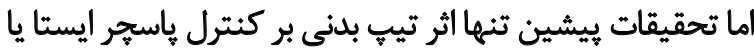

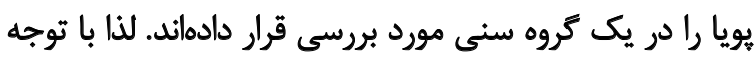

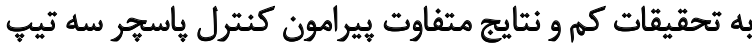

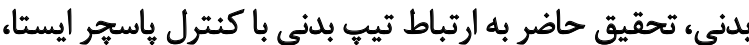
نيمهيوياو يويا يرداخته است.
است [ب]. برخى مطالعات به اثرات افزايش وزن بر كنترل قامتى

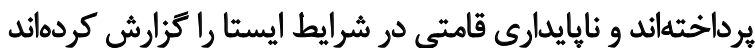

$[\mathrm{Y}-\mathrm{V}]$

كوربيل از يك مدل هاسكمانى براى مطالعه اثر وزن بدن

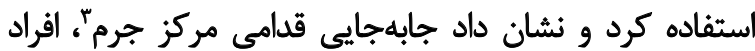

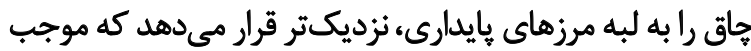

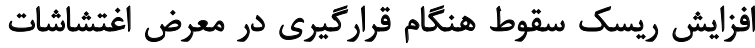

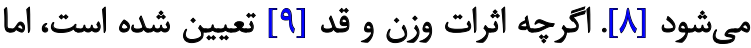

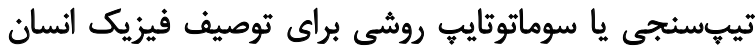

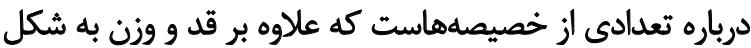

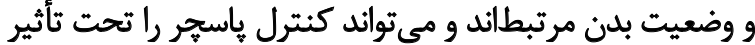

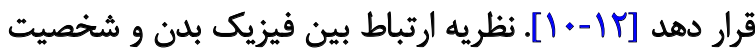

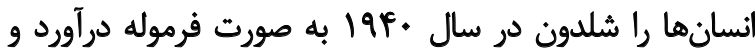

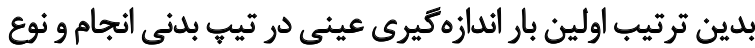

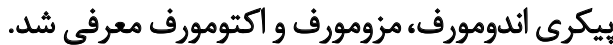

نخستين جزء يا اندومورفى، ميزان جاقى نسبى بدن ان راه

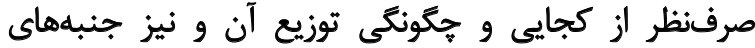

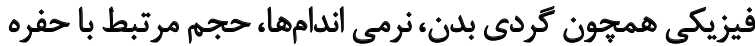

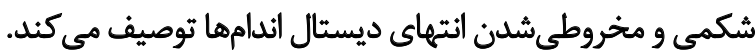

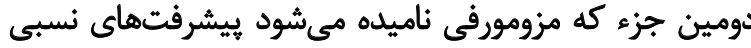

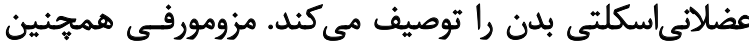

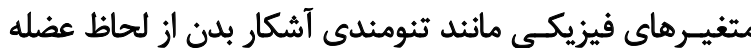

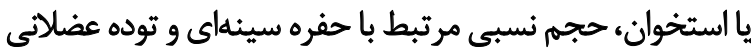

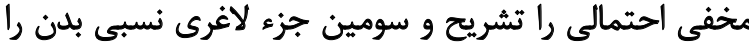

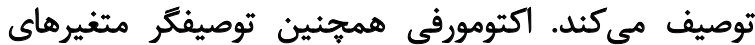

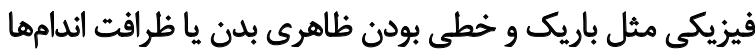

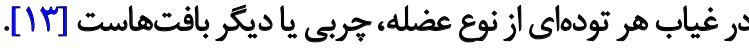

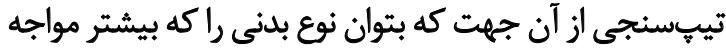

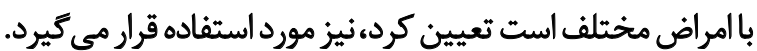

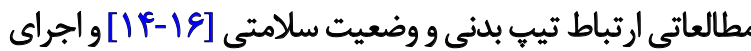

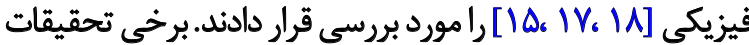

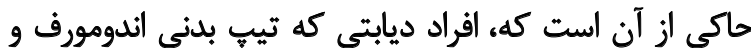

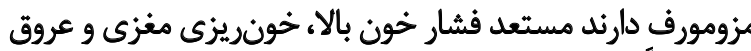

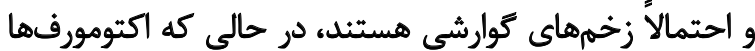

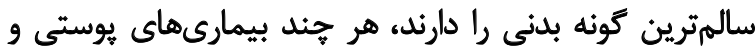

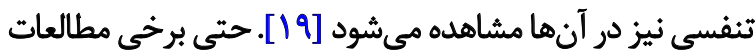

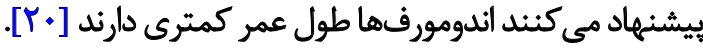
انقباض عضلات، مسئول اصلى حفظ پِاسجر در هر دو حالت

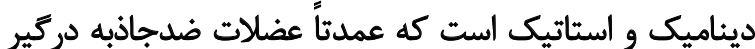

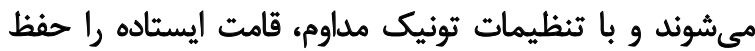

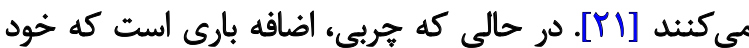

3. Center of mass 
يكى از آزمونهاى عملكردى برآورد كنترل ياسجير يويا و نيز

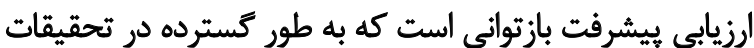

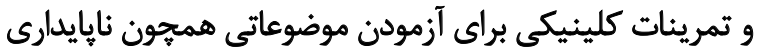

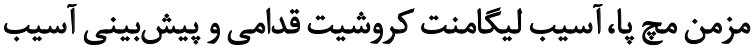

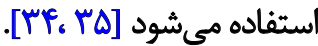

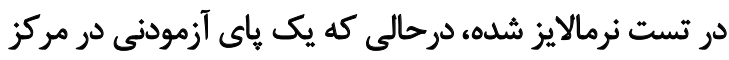

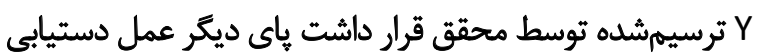

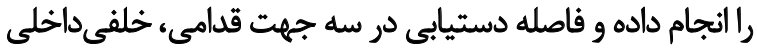
و خلفى خارجى اندازهكيرى شد. براى كاهش اثر ئر يادكيرى تنهيا

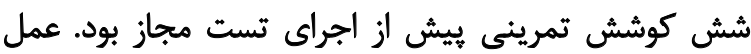

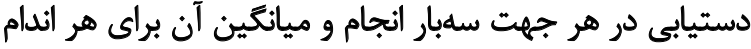

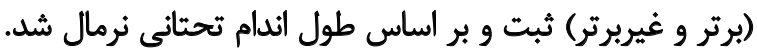

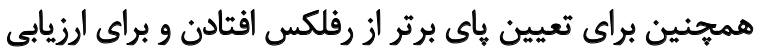

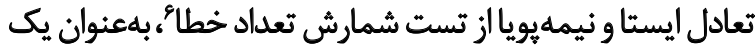

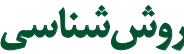

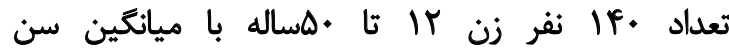

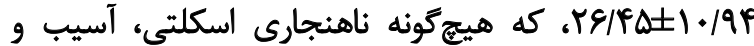

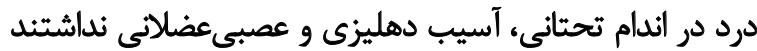

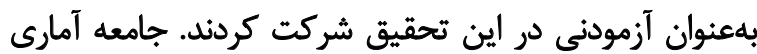

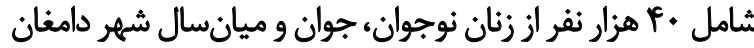

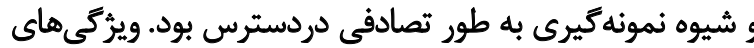

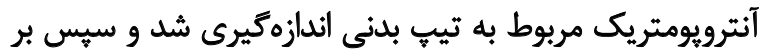

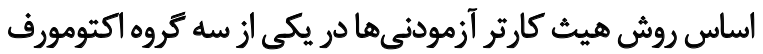

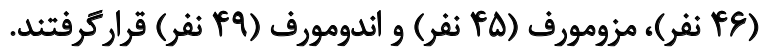

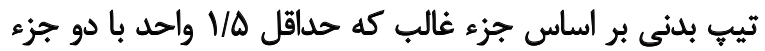

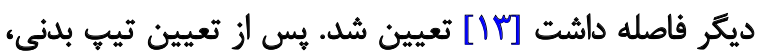

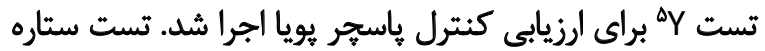

6. BESS

5. Y balance test

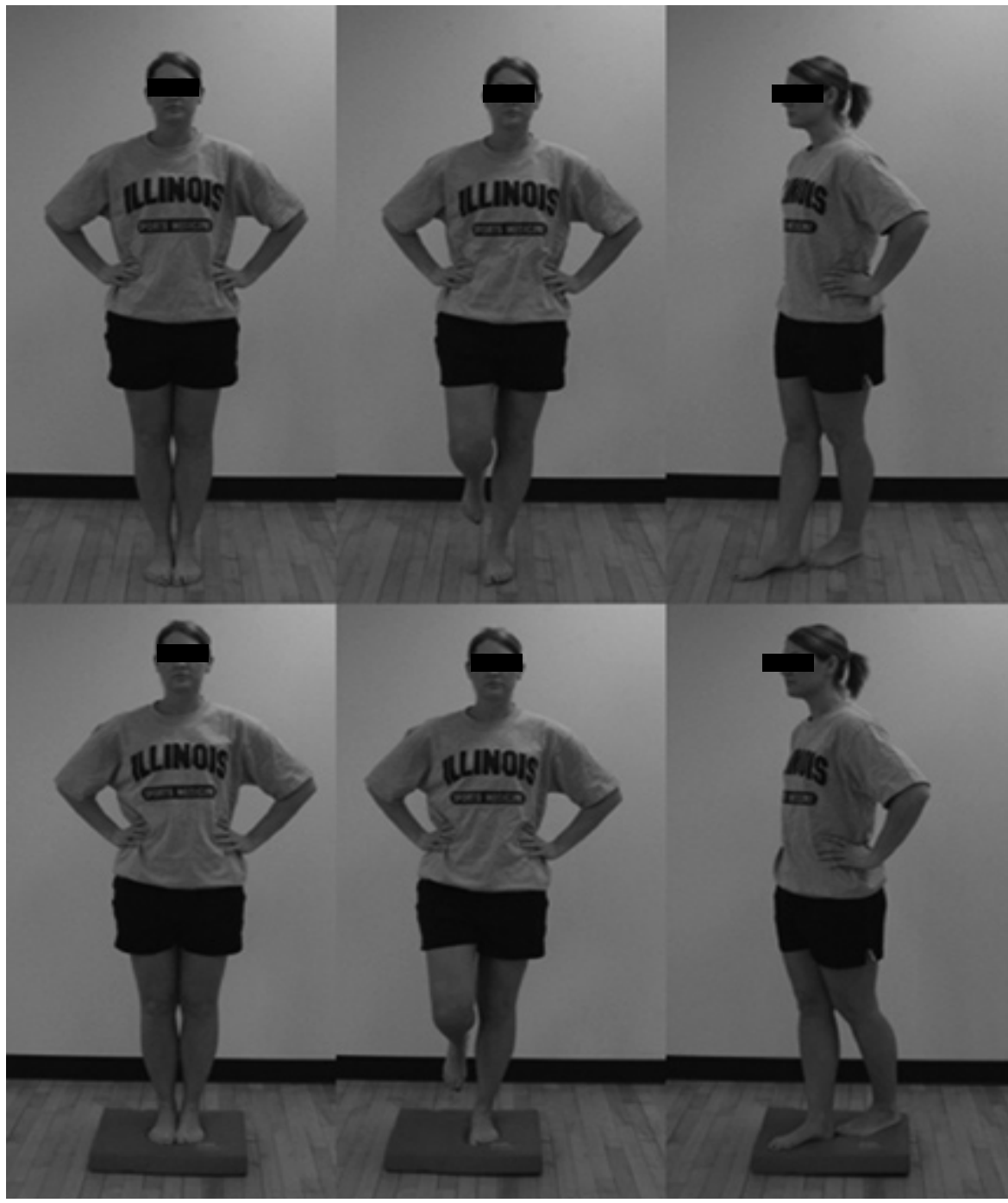


جدول ا. جدول مثتقاطع كنترل ياسجر ايستا و نيمهيويا

\begin{tabular}{|c|c|c|c|c|c|c|}
\hline \multirow{2}{*}{ 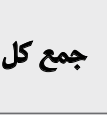 } & \multicolumn{3}{|c|}{ تيبي بدنى } & \multirow{2}{*}{ فرواوانى فر فراوانى } & \multirow{2}{*}{ تعداد خطا } & \multirow{2}{*}{ كتترل ياسجر } \\
\hline & اندومورف & مزومورف & اكتومورف & & & \\
\hline H & 9 & ri & Ir & تعداد & \multirow{2}{*}{$<r$} & \\
\hline$r+/ r$ & Wt & $r E / Y$ & rNr & درصد در بين تيبي بلنى & & \\
\hline rY & $\checkmark$ & 1. & 1. & تعداد & \multirow{2}{*}{$r-8$} & \\
\hline $19 / r$ & $1 \in / 4$ & $M / r$ & $M / N$ & درصد در بين تئي بلدئي & & \\
\hline r & $\gamma$ & $r$ & $p$ & تعداد & \multirow{2}{*}{$8-9$} & \\
\hline $9 / \pi$ & $\mid F / \pi$ & $\varphi / f$ & NV & درصل در بين ثيب بلنى & & \\
\hline$r$. & 9 & $r$ & 9 & تعداد & \multirow{2}{*}{$9-11$} & ايستا \\
\hline $1 F / \pi$ & We & $e / f$ & $19 / 8$ & درصل در بين تيبي بلنئي & & \\
\hline$r \cdot$ & 9 & 8 & $\Delta$ & تعداد & \multirow{2}{*}{$1 r-10$} & \\
\hline $\mid \varphi / r$ & We & $1 w / r^{m}$ & $1 . / 9$ & درصد در بين ثيب بدنى & & \\
\hline IV & $\wedge$ & $\varphi$ & $\Delta$ & تعداد & \multirow{2}{*}{$>10$} & \\
\hline $\mid r / 1$ & $\mid s / \mu$ & Na & $1 . / 9$ & درصد در بين ثيب بدنى & & \\
\hline If. & $p q$ & Fa & te & تعداد & \multirow{2}{*}{\multicolumn{2}{|c|}{ جمع كل }} \\
\hline $1.0 \%$ & $1 .+1$ & 1.01 & $1.0 \%$ & درصل در بين تيبي بلثئي & & \\
\hline 81 & M & $r \cdot$ & $r$ & تعداد & \multirow{2}{*}{$<1$. } & \\
\hline FNE & reN & $\operatorname{se/N}$ & $\mu r / \Delta$ & درصد در بين تيب بدنى & & \\
\hline Ar & M & 1. & $r+$ & تعداد & \multirow{2}{*}{$1+-r}$. & \\
\hline $\mathrm{r} / \mathrm{I}$ & $19 \pm / 9$ & $M / K$ & $P r / \Delta$ & درصل در بين تيبي بلنى & & نسملويا \\
\hline 18 & $r$ & $\Delta$ & f & تعداد & \multirow{2}{*}{$r \cdot-r \cdot$} & 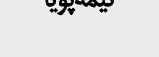 \\
\hline $11 / 9$ & $\mid \varphi / r$ & $11 / 1$ & $N Y$ & درصد در بين تيبي بدنى & & \\
\hline r & $r$ & $\cdot$ & $r$ & تعداد & \multirow{2}{*}{$>r}$. & \\
\hline$r / 9$ & $\% /$ & $\%$ & $F / T$ & درصد در بين تيب بدنى & & \\
\hline If. & $p q$ & PQ & ie & تعداد & \multirow{2}{*}{\multicolumn{2}{|c|}{ جمع كل }} \\
\hline $1 . . \%$ & $1 . . \%$ & $1 . .1$. & $1.0 \%$ & درصل در بين تيب بدئى & & \\
\hline
\end{tabular}

\section{مجله بيومكانيك ولنث}

يويا (فاصله دستيابى در سه جهت آزمون Y) از آمـار توصيفى و

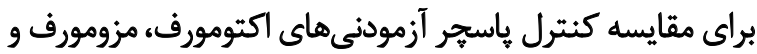

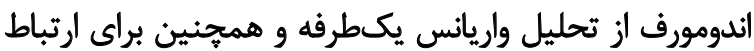

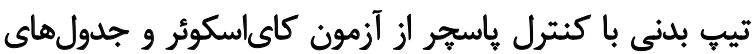

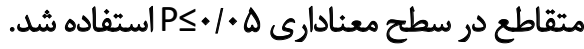

ثتايج

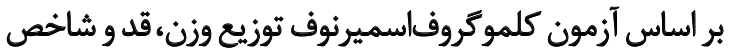

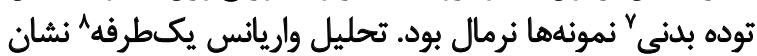

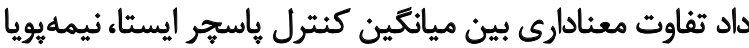

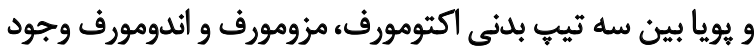

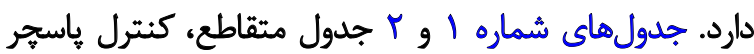
بر اساس تيڤ بدنى را نشان مي دهد. FE/V درصد مزومورفها،

7. Body Mass Index (BMI) 8. ANOVA
تست ميدانى معتبر استفاده شد [عب]. در اين روش، آزمودنى در

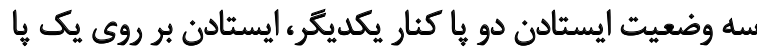

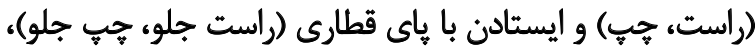

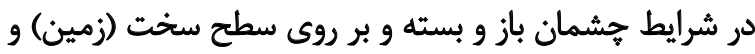

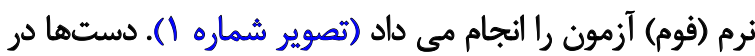

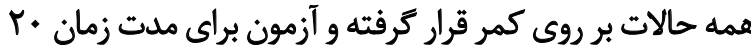

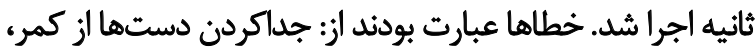

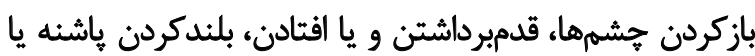

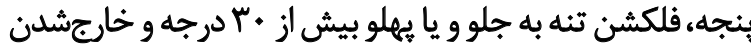

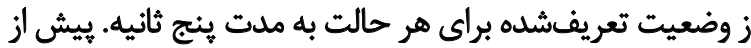

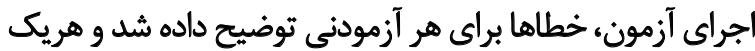

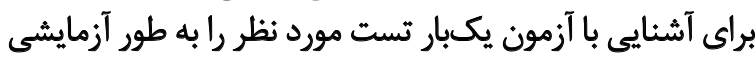
اجراكردند.

براي محاسبه ميانكَين و انحراف استاندارد سن، قد، وزن و وزئو كثترل ياسجر ايستا، نيمهيويا (تعداد خطاهاى آزمون BESS) و 
جدول r. جدول متثاطع كنترل ياسجر بويا

\begin{tabular}{|c|c|c|c|c|c|c|}
\hline \multirow{2}{*}{ جمع كل } & \multicolumn{3}{|c|}{ تيبِبدنى } & \multirow{2}{*}{ درواواتى } & \multirow{2}{*}{ تعد|د خُطا } & \multirow{2}{*}{ كثترل باسيجر } \\
\hline & اندومورف & مزومورف & اكتومورف & & & \\
\hline
\end{tabular}

Tr

درصد در بين تيبي بلنى

$<\Delta Q$

SQ $\quad$ Tl If

درصد در بين تيبي بلدنى

$\Delta Q-8 \Delta$

HT $\quad M \quad$ it

درصد در يين تيبي بلنى

قدامى

$8 \quad 1 \quad 00000$

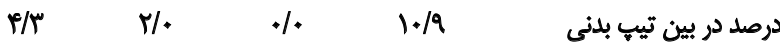

If. Fq pa pq iq ita

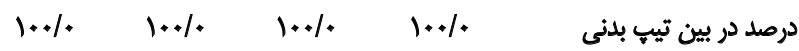

جمع كل

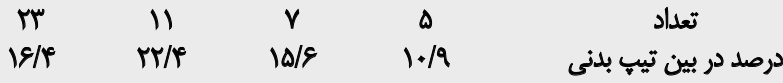

Ye $1010 \quad 10$

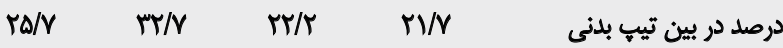

$\Delta 9 \quad 19 \quad 19 \quad M \quad$ TI

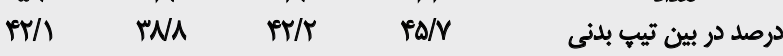

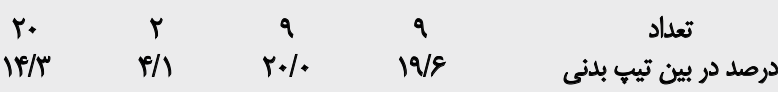

$\begin{array}{lllll}r & 1 & \end{array}$

درصد در بين تييه بدنى

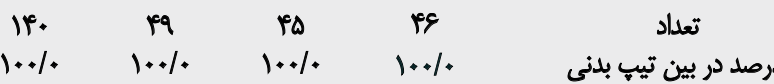

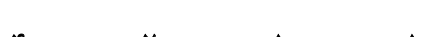

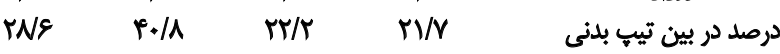

تعلياد

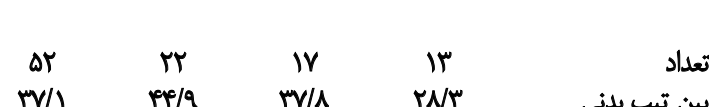

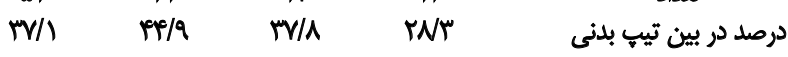

تE

درصد در بين تيبي بدنى

خلفىداخلى

8 ت 1000

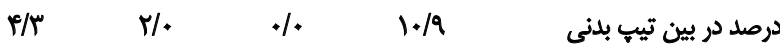

$>\wedge \Delta$

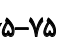




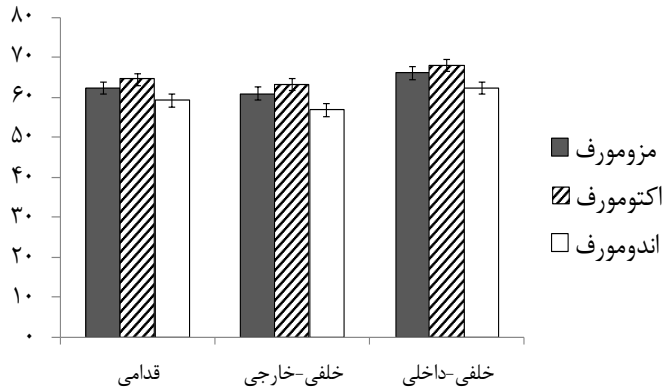

مجله بيومكانيك ورنش

تصوير ب. كتبرل ياسجر يويا (ميانكين ميزان دستيابى)

آزمون كاى اسكوئر نشان داد گروه مزومورف بهترين و اندومورفها

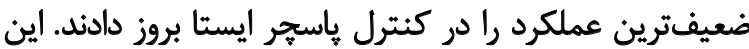

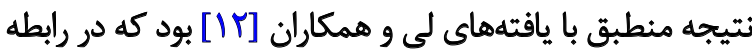

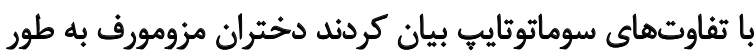

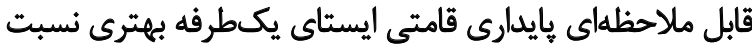

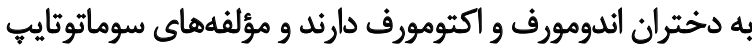

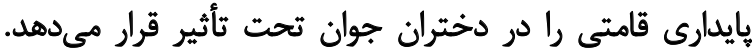

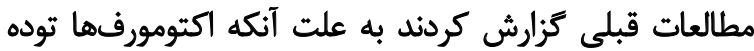

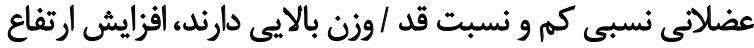

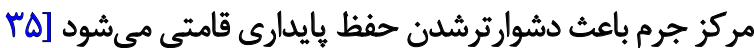

[TFF。

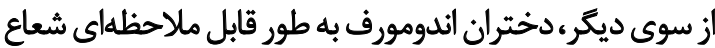

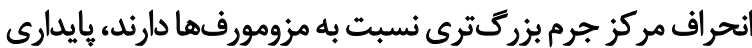

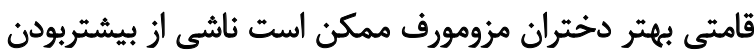

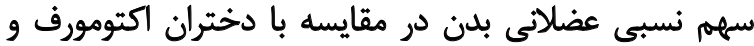

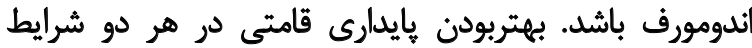

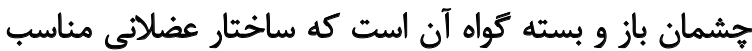

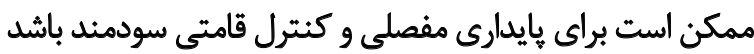

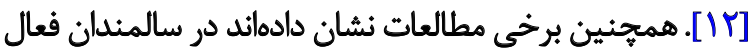

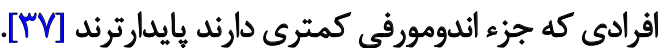
نتايج بهدست آمده در مورد بدتربودن كنترل قامتى اندومورفها

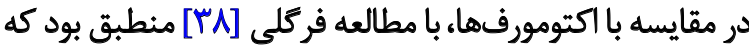

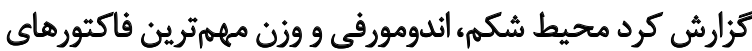

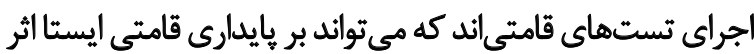

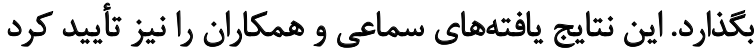

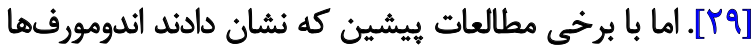

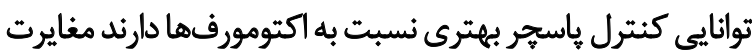

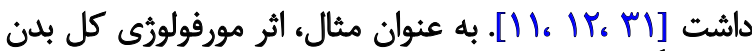

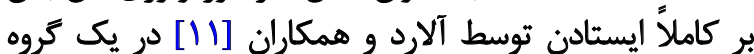

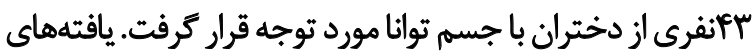

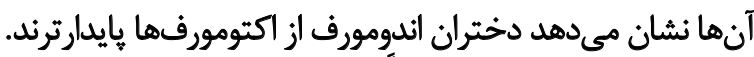
تفاوتهاي مشاهدهده احتمالأمىتواند مربوط به سن سن نمونه هاني

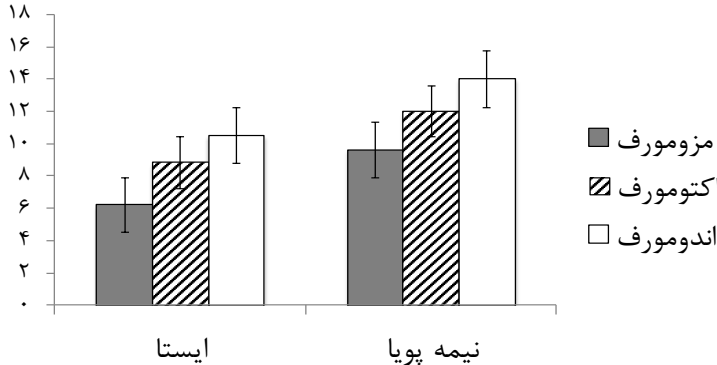

مجله بيومكانيك ورنش

تصوير Y. كنترل ياسجر ايستاو نيمهيويا (ميانكين تعداد خطا)

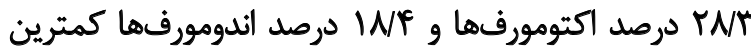

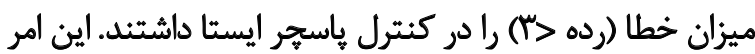

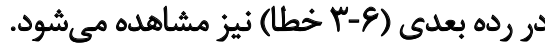

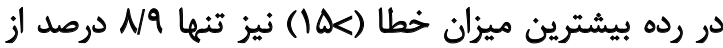

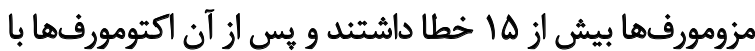

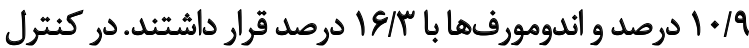

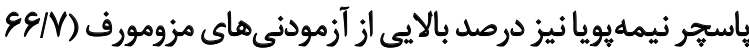

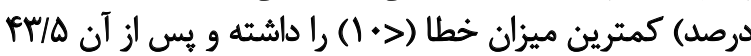

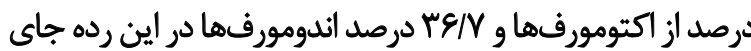
كرفتند (جدول شماره ()).

تفاوت سه تيب بدنى در جهت قدامى تست Y معنادار و و

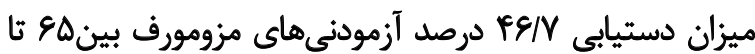

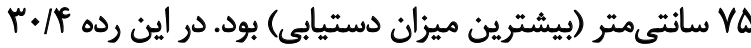

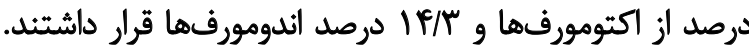

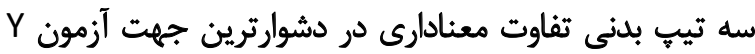

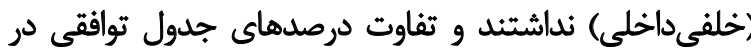

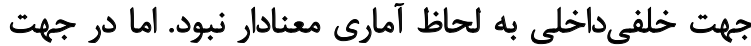

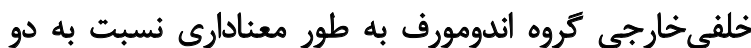

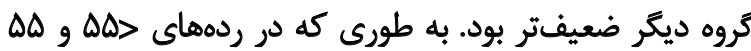

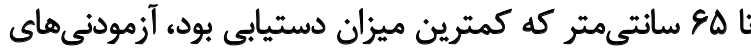

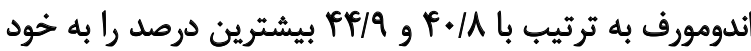

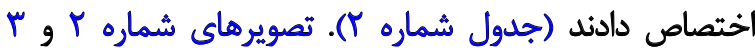

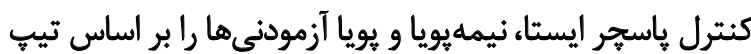
يلني نشان مي دهل Hes?

هدف از اين تحقيق ارتباط تيبّ بدنى با كنترل ياسجر ايستا،

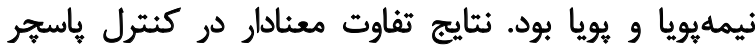

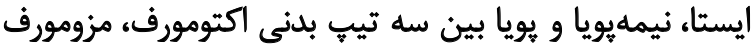

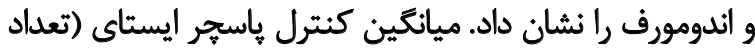

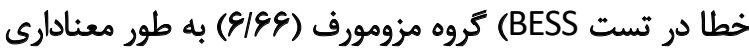

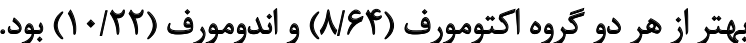




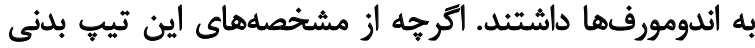

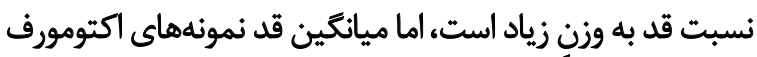

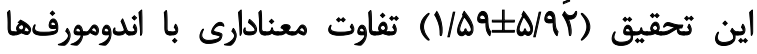

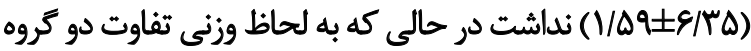

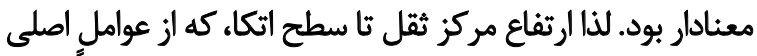

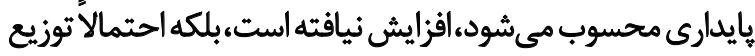

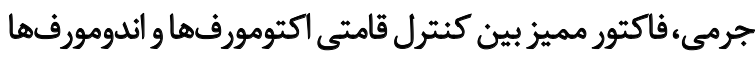
بوده است.

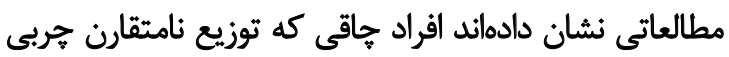

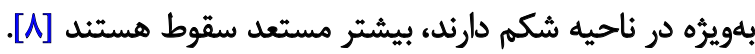

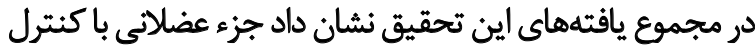

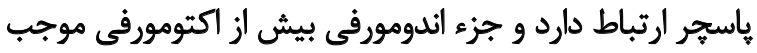

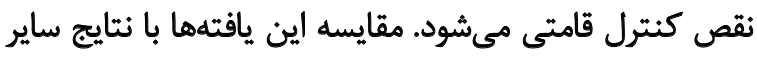

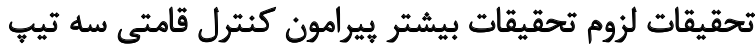

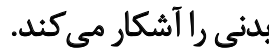

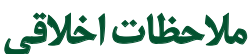 \\ يبروى از اصول اخلاق ئروهش}

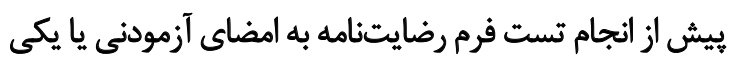

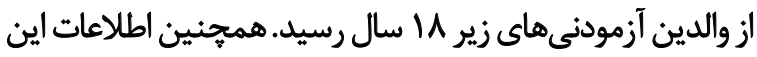

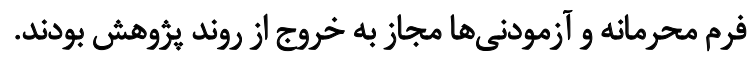

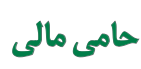

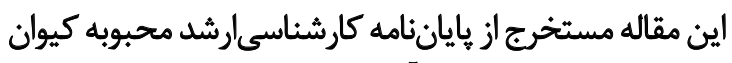

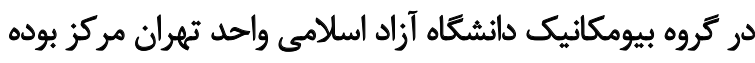

$$
\text { مشاركت نويسندئان }
$$

مفهومسازى، روششناسى، اعتبارسنجى، تحليل، تحقيق

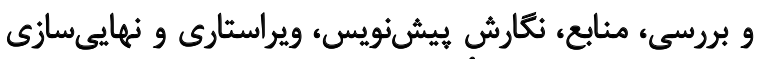

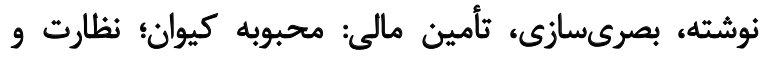

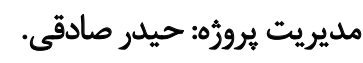

$$
\text { تعارض منافع }
$$

هيجزونـه تعـارض احتمالى منافـع در ايـن بثروهـش وجـود

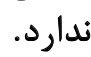

اين مطالعات باشد.

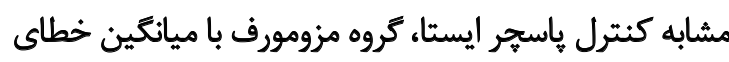

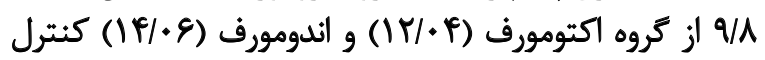

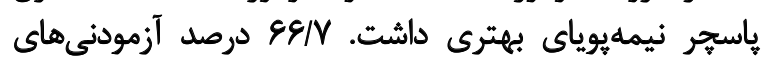

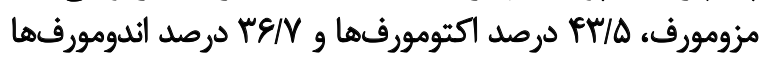

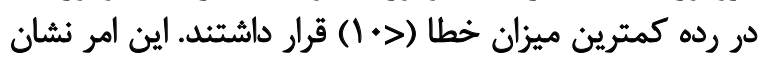

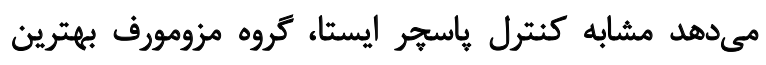

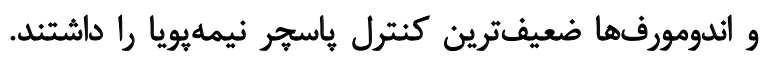

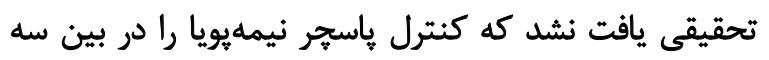

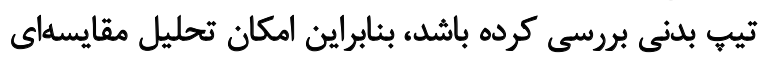

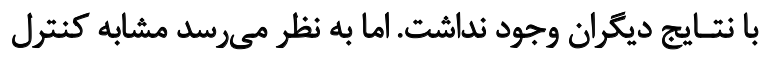

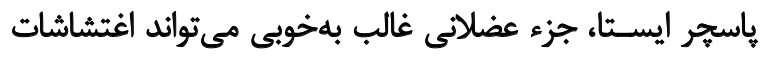

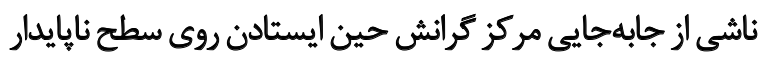

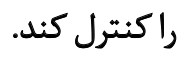
كنترل پاسجر يويا (ميانكين فاصله دستيابى تست (Y) بين

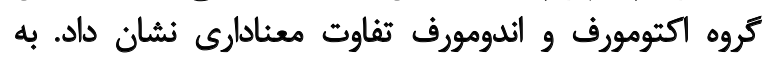

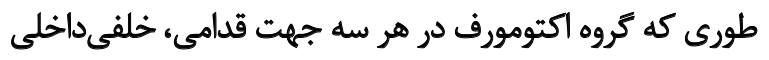

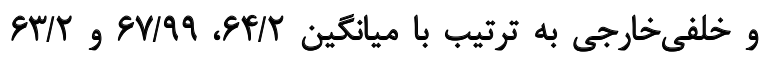

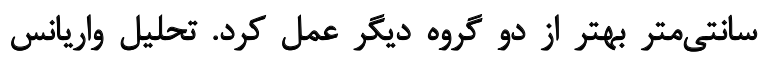

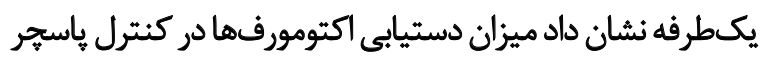

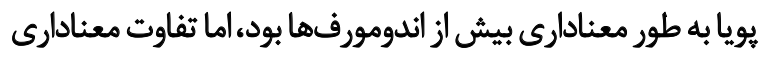

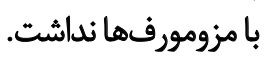

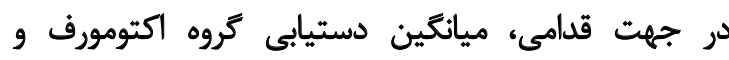

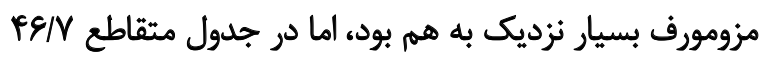

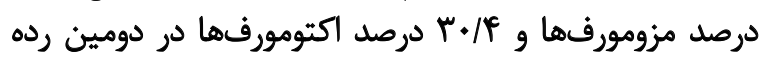

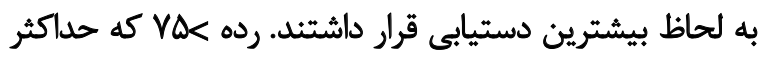

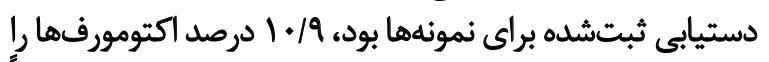

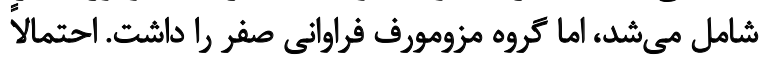

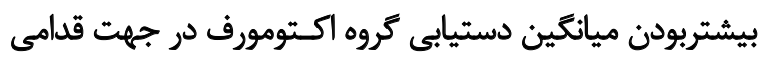

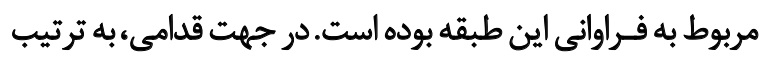

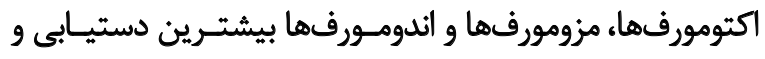

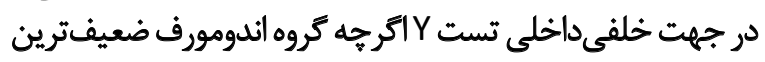

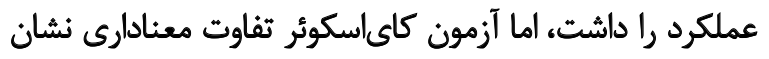

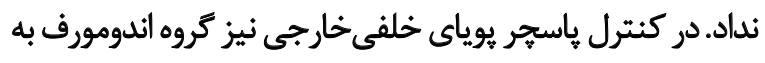

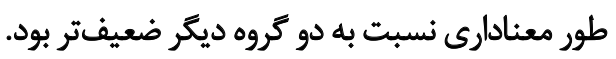

\section{نتيجليَيرىنهايى}

به طور كلى نتايج حاكى از عملكرد بهتر مزومورفها و و بوري

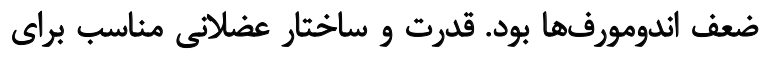

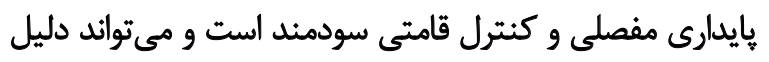

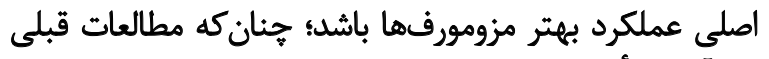

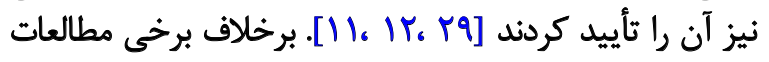

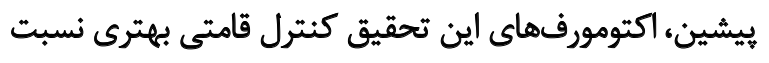




\section{References}

[1] Ivanenko Y, Gurfinkel VS. Human postural control. Frontiers in Neuroscience. 2018; 12:171. [DOI:10.3389/fnins.2018.00171] [PMID] [PMCID]

[2] Hue O, Simoneau M, Marcotte J, Berrigan F, Doré J, Marceau P, et al. Body weight is a strong predictor of postural stability. Gait \& Posture. 2007; 26(1):32-8. [DOI:10.1016/j.gaitpost.2006.07.005] [PMID]

[3] Błaszczyk JW, Cieślinska-Swider J, Plewa M, Zahorska-Markiewicz B, Markiewicz A. Effects of excessive body weight on postural control. Journal of Biomechanics. 2009; 42(9):1295-300. [DOI:10.1016/j.jbiomech.2009.03.006] [PMID]

[4] Maffiuletti NA, Agosti F, Proietti M, Riva D, Resnik M, Lafortuna CL, et al. Postural instability of extremely obese individuals improves after a body weight reduction program entailing specific balance training. Journal of Endocrinological Investigation. 2005; 28(3):2-7. [DOI:10.1007/ BF03345521] [PMID]

[5] Villarrasa-Sapiña I, Álvarez-Pitti J, Cabeza-Ruiz R, Redón P, Lurbe E, García-Massó X. Relationship between body composition and postural control in prepubertal overweight/obese children: A cross-sectional study. Clinical Biomechanics. 2018; 52:1-6. [DOI:10.1016/j.clinbiomech.2017.12.010] [PMID]

[6] Handrigan GA, Corbeil P, Simoneau M, Teasdale N. Balance control is altered in obese individuals. Journal of Biomechanics. 2010; 43(2):3834. [DOI:10.1016/j.jbiomech.2009.08.041] [PMID]

[7] Villarrasa-Sapiña I, García-Massó X, Serra-Añó P, Garcia-Lucerga C, Gonzalez LM, Lurbe E. Differences in intermittent postural control between normal-weight and obese children. Gait \& Posture. 2016; 49:1-6. [DOI:10.1016/j.gaitpost.2016.06.012] [PMID]

[8] Corbeil P, Simoneau M, Rancourt D, Tremblay A, Teasdale N. Increased risk for falling associated with obesity: Mathematical modeling of postural control. IEEE Transactions on Neural Systems and Rehabilitation Engineering. 2001; 9(2):126-36. [DOI:10.1109/7333.928572] [PMID]

[9] Berger W, Trippel M, Dicsher M, Dietz V. Influence of subjects' height on the stabilization of posture. Acta Oto-Laryngologica. 1992; 112(1):22-30. [DOI:10.3109/00016489209100778] [PMID]

[10] Allard P, Nault ML, Hinse S, LeBlanc R, Labelle H. Relationship between morphologic somatotypes and standing posture equilibrium. Annals of Human Biology. 2001; 28(6):624-33. [DOI:10.1080/03014460110047946] [PMID]

[11] Farenc I, Rougier P, Berger L. The influence of gender and body characteristics on upright stance. Annals of Human Biology. 2003; 30(3):27994. [DOI:10.1080/0301446031000068842] [PMID]

[12] Lee AJY, Lin WH. The influence of gender and somatotype on single-leg upright standing postural stability in children. Journal of Applied Biomechanics. 2007; 23(3):173-9. [DOI:10.1123/jab.23.3.173] [PMID]

[13] Duquet W, Carter JEL. Somatotyping. In: Eston R, Reilly T, editors. Kinanthropometry and Exercise Physiology Laboratory Manual: Tests, Procedures and Data. London: Taylor \& Francis; 1996.

[14] Mozumdar A, Roy SK. Somatotype of the individuals with lower extremity amputation and its association with cardiovascular risk. Anthropologischer Anzeiger. 2008; 66(1):99-116. [DOI:10.1127/ aa/66/2008/99] [PMID]

[15] Marta C, Marinho DA, Costa AM, Barbosa TM, Marques MC. Somatotype is more interactive with strength than fat mass and physical activity in peripubertal children. Journal of Human Kinetics. 2011; 29A:83-91. [DOI:10.2478/v10078-011-0063-4] [PMCID]
[16] Singh SP. Somatotype and disease - A review. Anthropologist. 2007; (3):251-61.

[17] Kolpakov VV, Bespalova TV, Bragin AV, Lebedeva KA, Tomilova EA, Vesnina TA. [The concept of typological variability of physiological individuality: II. Somatotype heterogeneity of population groups differing in habitual physical activity (Russian)]. Fiziologiia Cheloveka. 2009; 35(1):75-83. [DOI:10.1134/S0362119709010113] [PMID]

[18] Janssen I, Heymsfield SB, Ross R. Application of simple anthropometry in the assessment of health risk: Implications for the Canadian physical activity, fitness and lifestyle appraisal. Canadian Journal of Applied Physiology. 2002; 27(4):396-414. [DOI:10.1139/h02-021]

[19] Calden G, Dupertuis W, Lewis W. Body types and tuberculosis. Psychosomatic Medicine. 1959; 21(6):460-72. [DOI:10.1097/00006842 195911000-00004]

[20] Damon A, Staff Fl, Hooton EA. Physique, longevity and number of offspring: Possible stabilizing selection in man, proceedings of the fortieth annual meeting Boston, April 15-17, 1971. American Association of Physical Anthropologists. 1971; 35(2):276. [DOI:10.1002/ ajpa.1330350220]

[21] Wuehr M, Kugler G, Schniepp R, Eckl M, Pradhan C, Jahn K, et al. Balance control and anti-gravity muscle activity during the experience of fear at heights. Physiological Reports. 2014; 2(2):e00232. [DOI:10.1002/ phy2.232] [PMID] [PMCID]

[22] Purenović-Ivanović T, Popović R, Bubanj S, Stanković R. Body composition in high-level female rhythmic gymnasts of different age categories. Science \& Sports. 2019; 34(3):141-8. [DOI:10.1016/j.scispo.2018.10.010]

[23] Pineda A, López J, Martinez C, Medina M. Somatotype and psychological profile of Mexican elite gymnasts. International Journal of Hispanic Psychology. 2011; 3(2):303-16.

[24] Wilson BR, Olson HW, Sprague HA, Van Huss WD, Montoye HJ. Somatotype and longevity of former university athletes and nonathletes. Research Quarterly for Exercise and Sport. 1990; 61(1):1-6. [DOI:10.1080/ 02701367.1990.10607472] [PMID]

[25] Mitchell RJ, Lord SR, Harvey LA, Close JCT. Associations between obesity and overweight and fall risk, health status and quality of life in older people. Australian and New Zealand Journal of Public Health. 2014 38(1):13-8. [DOI:10.1111/1753-6405.12152]

[26] Pizzigalli L, Micheletti Cremasco M, Mulasso A, Rainoldi A. The contribution of postural balance analysis in older adult fallers: A narrative review. Journal of Bodywork and Movement Therapies. 2016; 20(2):40917. [DOI:10.1016/j.jbmt.2015.12.008] [PMID]

[27] Trombetti A, Reid KF, Hars M, Herrmann FR, Pasha E, Phillips EM, et al. Age-associated declines in muscle mass, strength, power, and physical performance: Impact on fear of falling and quality of life. Osteoporosis International. 2016; 27(2):463-71. [DOI:10.1007/s00198-015-3236-5] [PMID] [PMCID]

[28] Bulbulian R, Hargan ML. The effect of activity history and current activ ity on static and dynamic postural balance in older adults. Physiology \& Behavior. 2000; 70(3-4):319-25. [DOI:10.1016/S0031-9384(00)00272-9]

[29] Samaei A, Bakhtiary AH, Hajihasani A. Endomorphs show higher postural sway than other somatotypes subjects. Middle East Journal of Rehabilitation and Health. 2014; 1(2):e23470. [DOI:10.17795/mejrh-23470]

[30] Kim J, Kwon Y, Chung HY, Kim CS, Eom GM, Jun JH, et al. Relationship between body factors and postural sway during natural standing. International Journal of Precision Engineering and Manufacturing. 2012; 13(6):963-8. [DOI:10.1007/s12541-012-0125-0] 
[31] Allard P, Chavet P, Barbier F, Gatto L, Labelle H, Sadeghi H. Effect of body morphology on standing balance in adolescent idiopathic scoliosis. American Journal of Physical Medicine \& Rehabilitation. 2004; 83(9):689-97. [DOI:10.1097/01.PHM.0000137344.95784.15] [PMID]

[32] Peterson ML, Christou E, Rosengren KS. Children achieve adult-like sensory integration during stance at 12-years-old. Gait \& Posture. 2006; 23(4):455-63. [DOI:10.1016/j.gaitpost.2005.05.003] [PMID]

[33] Lebiedowska MK, Syczewska M. Invariant sway properties in children Gait \& Posture. 2000; 12(3):200-4. [DOI:10.1016/S0966-6362(00)000801]

[34] Patel L, Sarkar B, Kumar P, Sahay P, Laha K, Sarkar N. Normative values of star excursion balance test in young adults: A cross sectional study. International Journal of Advanced Research. 2018; 6(8):206-14. [DOI:10.21474/IJAR01/7512]

[35] Gribble PA, Hertel J, Plisky P. Using the star excursion balance test to assess dynamic postural control deficits and outcomes in lower extremity injury: A literature and systematic review. Journal of Athletic Training. 2012; 47(3):339-57. [DOI:10.4085/1062-6050-47.3.08] [PMID] [PMCID]

[36] Bell DR, Guskiewicz KM, Clark MA, Padua DA. Systematic review of the balance error scoring system. Sports Health: A Multidisciplinary Approach. 2011; 3(3):287-95. [DOI:10.1177/1941738111403122] [PMID] [PMCID]

[37] Daniels SR. The consequences of childhood overweight and obesity. The Future of Children. 2006; 16(1):47-67. [DOI:10.1353/foc.2006.0004] [PMID]

[38] Fregly AR, Oberman A, Graybiel A, Mitchell RE. Thousand aviator study: Nonvestibular contributions to postural equilibrium functions. Aerospace Medicine. 1968; 39(1):33-7. 